ICNMM2009-82071

\title{
LASER-INDUCED FLUORESCENCE DETECTION OF COPPER ION WITH A RHODAMINE DERIVATIVE BY A CAPILLARY ELECTROPHORESIS MICROCHIP
}

\author{
Yong Yu1, Yeng Yang ${ }^{12}$, Feng Shen ${ }^{1}$ \\ 1 National Yicrogravity Lab, Institute of Yechanics, Chinese Academy of Science, Beijing 100190 \\ 2 College of Chemistry, Beijing Normal University, Beijing 100875 \\ E-mail: yuyonglimech. ac. cn
}

\section{ABSTRACT}

A capillary electrophoresis microchip system with a confocal LIF detector was successfully constructed. $\mathrm{RBPhOH}$, a new fluorescence dye synthesized from rhodamine B, was first investigated in a glass microchip for the selective and sensitive determination of copper ion by LIF method. The influences of the $\mathrm{pH}$ value, concentration of running buffer, detection voltage, and sample loading time on the response of LIF detector were studied. $\mathrm{RBPhOH}$ dissolved in $20 \mathrm{mM} \mathrm{pH}$ 6.0 Tris containing $10 \%$ acetonitrile could detect copper ions with a detection limit of $1.34 \times 10^{-7} \mathrm{~mol} \mathrm{~L}^{-1}(\mathrm{~S} / \mathrm{N}=3)$, an order of magnitude lower than those of conductivity detectors.

\section{INTRODUCTION}

Micrototal analysis systems ( $\mu$ TAS) integrated some analytical procedures, including sample treatment, reaction, separation, detection et al, into single devices to fulfill the analysis and detection. Owing to their high degree of integration, portability, minimal reagent consumption, high performance and speed, the capillary electrophoresis microchips have drawn more and more attention. For decades, they have been popular analytical equipment for biomedical and pharmaceutical analysis, clinical diagnostics, environmental monitoring, forensic investigations, etc. Thus, Lab-on-a-chip was remarked as "a technology for this century" by Nature [1].

In microchip devices, the two principal methods for detection are optical and electrochemical. Electrochemical methods mainly contain conductive[2-6], amperometric[7-8], and potential means. However, the low sensitivity and repeatability limit their applications. The optical methods have higher sensitivity and better reproduction than electrochemical methods. Thus, there are some advantages in combining optical components with microchip devices. Among optical methods, Laser-induced Fluorescence (LIF) detection is a highly sensitive detection method in microchip systems. Yet, studies in the detection of metal ions in microchips by LIF have not been reported as far as authors knew. The optical arrangement of detection devices played an important role in the high sensitivity and low detection limit. There are typically two major types of arrangements, the confocal system and nonconfocal system [9-10]. Compared with the latter, the confocal LIF system exhibited higher sensitivity and lower LOD in separation and detection.

$\mathrm{Yu}$ Xiang et al [11] synthesized a kind of derivative compound from rhodamine $\mathrm{B}$, named $\mathrm{RBPhOH}$ for short, to analyze the $\mathrm{Cu}^{2+}$ by fluorescence. The initial goal of our research was to construct a microchip system with an LIF detector, and demonstrate the applicability of $\mathrm{RBPhOH}$ to the microchip for the selective and sensitive determination of $\mathrm{Cu}^{2+}$. This attempt could not only advance the sensitivity of detection of metal ions implemented in microchips, but also enlarge the fields of application of LIF detection method.

\section{REAGENTS AND SOLUTIONS}

$\mathrm{RBPhOH}\left(\mathrm{C}_{35} \mathrm{~N}_{4} \mathrm{O}_{3} \mathrm{H}_{36}, 560 \mathrm{~g} \mathrm{~mol}^{-1}\right)$ was provided by Professor Aijun Tong of Tsinghua University. Copper (II) nitrate, ferrous sulfate, ferric nitrate, chromic (III) sulfate, cadmium chloride, lead nitrate, mercuric chloride and acetonitrile were purchased from Beijing Chemical Reagents Company. All chemicals were analytical grade and used without any further purification. The running buffer solution for detection was prepared by Tris, $\mathrm{pH}$ 6.0. High-purity deionized water $(18.2 \mathrm{M} \Omega)$ was obtained by passing distilled water through a Milli-Q Plus water purification system. Stock solutions of metal ions $\left(\mathrm{Cu}^{2+}, \mathrm{Fe}^{2+}, \mathrm{Fe}^{3+}, \mathrm{Cr}^{3+}, \mathrm{Cd}^{2+}, \mathrm{Pb}^{2+}, \mathrm{Hg}^{2+}\right)$ were prepared by dissolving the corresponding salts in the run buffer. Aqueous solutions containing metal ion were mixed with the acetonitrile solution of $\mathrm{RBPhOH}$, and then diluted by running solution to prepare sample solution containing $10 \%$ 
acetonitrile. All solutions for electrophoresis experiments were filtered through a $0.2 \mu \mathrm{m}$ membrane filter before use.

\section{APPARATUS}

A 532-nm semiconductor laser (Output Power 50mw, Beijing lambdapro technologies Ltd, Beijing, China) was used as light source. The excitation laser power was adjusted by an attenuation filter to prevent not overly saturated photon emission from fluorophores. The laser beam was passed through a 532-nm band-pass filter (Shenyang HB Optical Technology Co., China), reflected by a mirror and focused by a $40 \times$ microscope objective lenses (0.65 NA, 2.95-mm-long working distance, Chongqing MIC Optical \& Electrical Instrument Co., Chongqing, China). Alignment of the microchip position was adjusted by an X-Y-Z translation stage (Beijing Optical Instrument Factory, Beijing, China). The excited fluorescence emissions from the sample were collected and focused by the same objective lenses, then successively transmitted through a 560-nm dichroic mirror (Beijing Film Machinery Research Institute, Beijing, China), a spatial filter (dia. $=500 \mu \mathrm{m})$ and three pieces of 560-nm long-pass filters (Beijing Film Machinery Research Institute, Beijing, China), into a photomultiplier tube (PMT-CR114, Hamamatsu, Japan), which transferred the fluorescence signal into the electric signal. The electric signal obtained by PMT was collected by a HP 34970A data acquisition unit (Agilent Technologies, Palo Alto, CA). Data treatment was performed in HP BenchLink DataLogger. A high voltage power supply with an adjustable voltage range from 0 to $+5000 \mathrm{~V}$ (The college of Chemistry, Chemical Engineering \& Material Science, Shandong Normal University, China) was used for sample injection and $\mathrm{CE}$ separation.

A cross-pattern glass microchip $(60 \mathrm{~mm}$ long $\times 20 \mathrm{~mm}$ wide $\times 2.2 \mathrm{~mm}$ deep) was purchased from Dalian Institute of Chemical Physics, CAS. The chip had a $45 \mathrm{~mm}$ long separation channel (from the cross to the buffer waste reservoir) and $10 \mathrm{~mm}$ long injection channel (between the sample and buffer reservoir). All channels were etched to a depth of $20 \mu \mathrm{m}$ and a width at the top of the channel of $60 \mu \mathrm{m}$. The glass chip was placed on a laboratory-built plastic holder for fixing the chip. Platinum wires electrodes were inserted into the reservoirs. Before measurement, the glass microchip was flushed with $0.1 \mathrm{M} \mathrm{NaOH}$, deionized water, and running buffer solution for 10 min each.

\section{RESULTS AND DISCUSSION}

The power of $\mathrm{RBPhOH}$ was gray color and it was difficult to dissolve in water but easily in acetonitrile. $\mathrm{RBPhOH}$ could react with copper ion and gave off fluorescence. The solution of $\mathrm{RBPhOH}$ in acetonitrile was colorless and transparent. After adding the aqueous solution of copper ion, it became claret. Even if the concentration of copper ion as low as $1 \times 10^{-6} \mathrm{~mol} \mathrm{~L}^{-}$ ${ }^{1}$, the color of mixed solution was still visible. The structures of reactant and its product were shown in Fig. 1

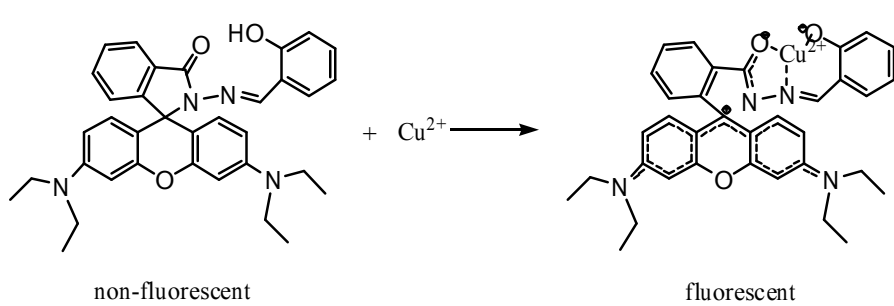

Fig. 1 The reaction between $\mathrm{RBPhOH}$ and a copper ion. Molecular structures of reactant and its product were optimized by DFT.

In a glass microchip, experiments were conducted by the confocal detection system. $3 \times 10^{-5}$ mol L $\mathrm{L}^{-1} \mathrm{RBPhOH}$ was used in the microchip because the weak fluorescence of $\mathrm{RBPhOH}$ itself couldn't be detected at this concentration. Experiments showed that the fluorescence intensity of the complex of $\mathrm{Cu}^{2+}$ with $\mathrm{RBPhOH}$ didn't be affected by adding $\mathrm{RBPhOH}$ into running buffer or not. Thus, $\mathrm{RBPhOH}$ was just added into sample solution.

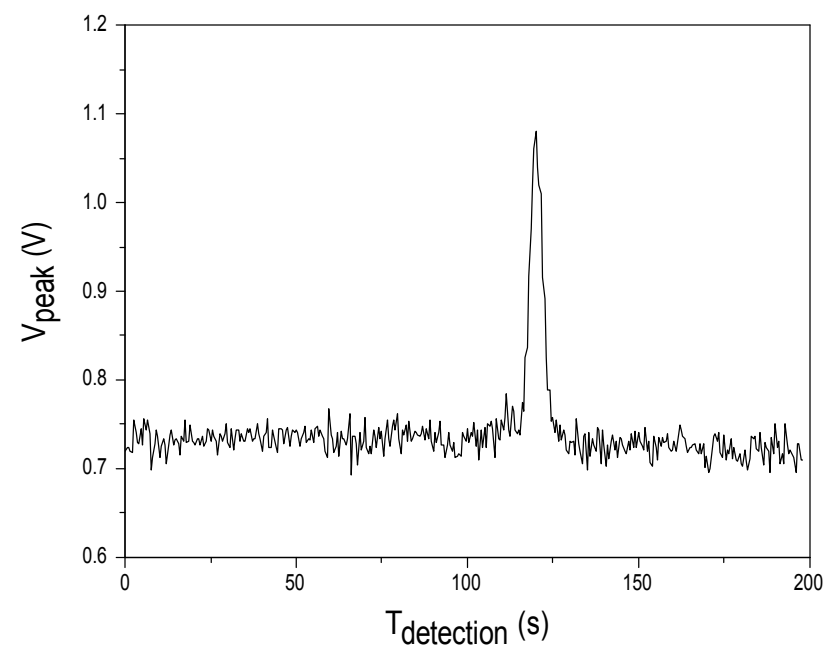

Fig. 2 Electropherograms of $3 \times 10^{-5} \mathrm{~mol} \mathrm{~L}^{-1} \mathrm{RBPhOH}$ in running buffer with $3.1 \times 10^{-6} \mathrm{~mol} \mathrm{~L}^{-1} \mathrm{Cu}^{2+}$. Running buffer: $20 \mathrm{mM} \mathrm{pH}=6$ Tris containing $10 \% \mathrm{CH}_{3} \mathrm{CN}$. Injection voltage: $400 \mathrm{~V}$; Sample loading time: 40 s; Detection voltage: $900 \mathrm{~V}$.

There was no obvious fluorescence detected from the sample solution containing $\mathrm{RBPhOH}$ without copper ions. When copper ions were added into sample solution, a fluorescent signal was clearly detected by the system, shown in Fig. 2. It was confirmed that LIF detection of copper ion with $\mathrm{RBPhOH}$ could be implemented in a glass microchip.

On the basis of experiments, a potential explanation for this phenomenon was put forward. This was because the association constant $(\mathrm{Ka})$ of copper ion reacted with $\mathrm{RBPhOH}$ was larger than other metal ions' [11]. In other word, copper ion combined with $\mathrm{RBPhOH}$ more strongly and the complex didn't dissociate easily. Yet, other metal ions may combine with $\mathrm{RBPhOH}$ weakly and therefore their complexes dissociated more easily in the separation channel. In conclusion, it was a selective and sensitive method to detect 
copper ions with $\mathrm{RBPhOH}$ in a microchip by an LIF detection system.

\section{LINEAR RANGE AND DETECTION LIIT}

According to the previous studies on the buffer $\mathrm{pH}$, concentration, the sample loading time and detection voltage, optimized conditions were $40 \mathrm{~s}$ sample loading time, $900 \mathrm{~V}$ detection voltage, $20 \mathrm{mM}$ pH6.0 Tris as running buffer. Under the selected conditions, a series of solutions of copper ions with a concentration range of $6.03 \times 10^{-7} \mathrm{~mol} \mathrm{~L}-1 \sim 1.56 \times 10^{-5} \mathrm{~mol} \mathrm{~L}^{-1}$ were detected by $\mathrm{RBPhOH}$. The regression analysis on calibration curve was 0.99969 . The detection limit for $\mathrm{Cu}^{2+}$ was evaluated to be $1.34 \times 10^{-7} \mathrm{~mol} \mathrm{~L} \mathrm{~L}^{-1}(\mathrm{~S} / \mathrm{N}=3)$. The $\mathrm{RSD}$ of migration time was $1.53 \%$.

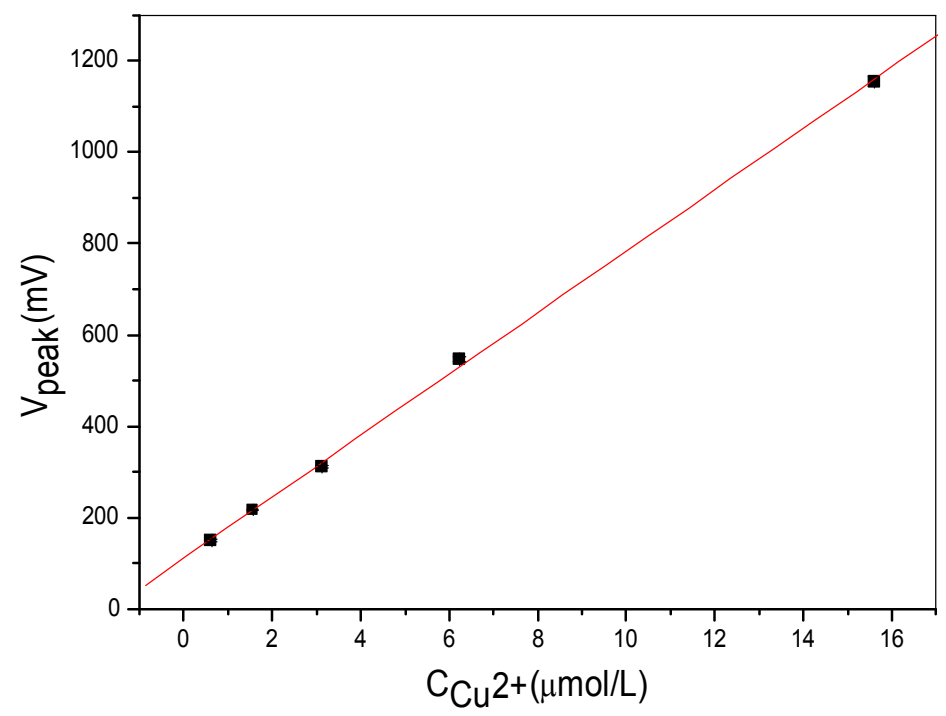

Fig. 3 The relationship between the peak height and the concentrations of copper ion. Experimental parameters: running buffer, 20mM pH6.0 Tris containing 10\% $\mathrm{CH}_{3} \mathrm{CN}$; Sample, $3 \times 10^{-5} \mathrm{~mol} / \mathrm{L} \mathrm{RBPhOH}$ and different concentrations of $\mathrm{Cu}^{2+}$ in running buffer. Injection voltage: $400 \mathrm{~V}$, injection time $40 \mathrm{~s}$; detection voltage, $900 \mathrm{~V}$.

\section{CONCLUSION}

We have demonstrated a rapid and facile method for selectively and sensitively detecting $\mathrm{Cu}^{2+}$ in glass microchips by a confocal LIF detector. As this method takes advantage of the sensitivity and wide availability of LIF detectors, this attempt could enlarge the applications of detecting poisonous heavy metal ions in microchips.

\section{ACXNOTLEDG ENTS}

The authors gratefully acknowledge the financial support provided to this study by the Innovation Fund of Chinese Academy of Science under Grant Nos. 0830082XM1. The authors also thank Prof. Aijun Tong of Tsinghua University for her kindness to give the fluorescence dye of $\mathrm{RBPhOH}$.

\section{REFERENCES}

1 R. Daw, J. Finkelstein, Nature, 2006, 442, 367.

2 M. Pumera, J. Wang, F. Opekar, I. Jelinek, J. Feldman, H. Lowe, S. Hardt, Anal. Chem., 2002, 74, 1968-1971.
3 J. Lichtenberg, N. F. de Rooij, E. Verpoorte, Electrophoresis, 2002, 23, 3769-3780.

4 S. Qu, X. H. Chen, D. Chen, P. Y. Yang, G. Chen, Electrophoresis, 2006, 27, 4910-4918.

5 C.Y. Lee, C.M. Chen, G.L. Chang, C.H. Lin, L.M. Fu, Electrophoresis, 2006, 27, 5043-5050.

6 J. Tanyanyiwa, P. C. Hauser, Anal. Chem., 2002, 74, 6378-6382.

7 X.A. Li, D.M. Zhou, J.J. Xu, H.Y. Chen, Talanta, 2007, 71, 1130-1135.

8 O. Chailapakul, S. Korsrisakul, W. Siangproh, K. Grudpan, Talanta, 2008, 74, 683-689.

9 X.M. Xu, L. Li, S. G. Weber, Trends Anal. Chem., 2007, 26, 68 .

10 J.L. Fu, Q. Fang, T. Zhang, X.H. Jin, Z.L. Fang, Anal. Chem., 2006, 78, 3827-3834.

11 Y. Xiang, A.J. Tong, P.Y. Jin, Y. Ju, Org. Lett., 2006, $8,2863-2866$. 